Nadine Martin

Chef de clinique, service de radiologie, hôpital Beaujon.

\section{L'imagerie par résonance magnétique en neuroradiologie}

\section{Quoique d'introduction récente, l'imagerie par résonance magnétique s'avère d'ores et déjà être l'une des méthodes neu- roradiologiques les plus performantes.}

\section{RÉFÉRENCES}

I. Zimmerman RA, Bilaniuk LT, Crossman RI et al. Cerebral tumors by partial saturation technique with resistive NMR. Neuroradiology 1985; 27 : 0-1 5

2. Kucharczyk W, Brant-Zawadzki M, Sobel MD. Central nervous system tumors in children: detection by magnetic resonance imaging. Radiology $1985 ; 155$ : $131-6$.

3. Lee BCP, Kneeland JB, Deck MD, Cahill PT. Posterior fossa lesions: Magnetic resonance imaging. Radiology 1984; 153 : 137-43.

4. Zimmerman RA, Bilaniuk LT, Packer R, et al. Resistive NMR of brain stem gliomas. Neuroradiology 1985; $27: 21-5$.

5. Claussen C, Laniado M, Kazner E, Schorner W, Felix R. Application of contrast agents in CT and MRI: their potential in imaging of brain tumors. Neuroradiology 1985; 27 (2) : 164-71.

6. Bocquet M, Vignaud J, Cosnard G, Ibazizen MT. NMR imaging of acoustic neurinoma. 7 . Neuroradiol 1984; 11 : 263-73.

7. Daniels DL, Schenck JF, Foster T, et al. Surface coil magnetic resonance imaging of the internal auditory canal. $A 7 R, 1985 ; 145: 469-72$.

8. New PJ, Bachow TB, Wismer GL, Rosen BR, Brady TJ. MR imaging of the acoustic nerves and small acoustic neuromas at $0,6 \mathrm{~T} . A 7 R, 1985$; 144 : $1021-6$.

9. Lee BCP, Deck MD. Sellar and juxtasellar lesions. Detection with MR. Radiology 1985; 157 : 143-7.

10. Bilaniuk LT, Zimmerman RA, Wehrli FW, et al. MRI of pituitary lesions at 1.0 to $1.5 \mathrm{~T}$ field strength. Radiology 1984; 153:415-8.

\section{ADRESSE}

N. Martin : Hôpital Beaujon, 100, boulevard du Général-Leclerc, 921 I 8 Clichy Cedex.

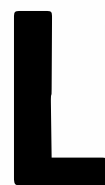

- imagerie par résonance magnétique (IRM), d'emblée orientée vers la pathologie ncuroencéphalique a déjà transformé la neuroradiologie. Sa détectabilité de contraste très supérieure à celle de la scanographie (ou tomodensitométrie, TDM), la différenciation aisée des structures cérébrales en particulier substance blanche-substance grise, l'excellente visibilité spontanée de la moelle jointe aux possibilités de vue tridimensionnelle permettent une vision anatomique jamais obtenue jusqu'alors. L'expérience de l'IRM commence à s'étendre. Ses indications se multiplient et se précisent, notamment vis-à-vis de la TDM.

\section{Pathologie encéphalique}

La pathologie encéphalique est très diverse : si l'IRM s'impose déjà très largement, son rôle n'apparaît cependant pas universel.

La pathologie tumorale : on doit ici différencier les tumeurs intra et extra-axiales. L'apport de l'IRM en pathologie tumorale intra-axiale, de type gliomateuse, est aujourd'hui acquis $[1,2,3]$ : sa sensibilité de détection apparaît en effet supérieure à celle du scanner. De façon globale, l'effet de masse sur les structures cérébrales et les citernes adjacentes est apprécié de manière quasi-constante quelles que soient les séquences utilisées. Surtout, la plupart des processus expansifs parenchymateux caractérisés par une augmentation de leurs temps de relaxation $\mathrm{T}_{\mathrm{I}}$ et $\mathrm{T}_{2}^{*}$ (voir Glossaire), sont aisément individualisés du parenchyme normal adjacent : selon les séquences utilisées, ils apparâitront hypointenses en inversion récupération (IR), mais hyperintenses sur les séquences en spin-echo (SE)*.

Si ces propriétés modifient peu le bilan des volumineux processus expansifs supratentoriels souvent de haut grade, très bien détectés par le scanner, elles prennent toute leur valeur dans les processus infiltratifs : isodenses en TDM, ces processus peuvent ne se manifester que par un effet de masse variable dont 


\section{* GLOSSAIRE*}

Spin: moment cinétique intrinsèque lié à une particule élémentaire.

Temps de relaxation $T_{1}$ (encore appelé temps de relaxation spin-réseau): après impulsion magnétique, temps de retour à l'état d'équilibre lié aux échanges d'énergie des spins avec leur environnement.

Temps de relaxation $T_{2}$ (encore appelé temps de relaxation spin -spin) : après impulsion magnétique, temps de retour à l'état d'équilibre lié aux échanges d'énergie entre les spins.

Le signal obtenu dépend à la fois de $T_{1}$ et $T_{2}$. Selon la séquence utilisée on privilégie le signal dépendant de $T_{1}$ ou de $T_{2}$. Les principales séquences utilisées sont :

Séquence de spin-écho: séquence d'impulsion permettant d'obtenir un signal IRM qui dépend de $T_{2}$.

Séquence de saturation partielle : séquence de spin-écho courte où le signal IRM obtenu est plutôt fonction de $T_{1}$.

Séquence pondérée en $T_{1} T_{2}$ : séquence dont le signal IRM obtenu est fonction de $T_{1}, T_{2}$.

Cisternotomodensitométrie:

examen TDM cérébral obtenu après opacification des citernes de la base par injection intra-thécale de produit de contraste.

Hyperintensité : signal IRM supérieur à la normale.

Hypointensité : signal IRM inférieur à la normale.

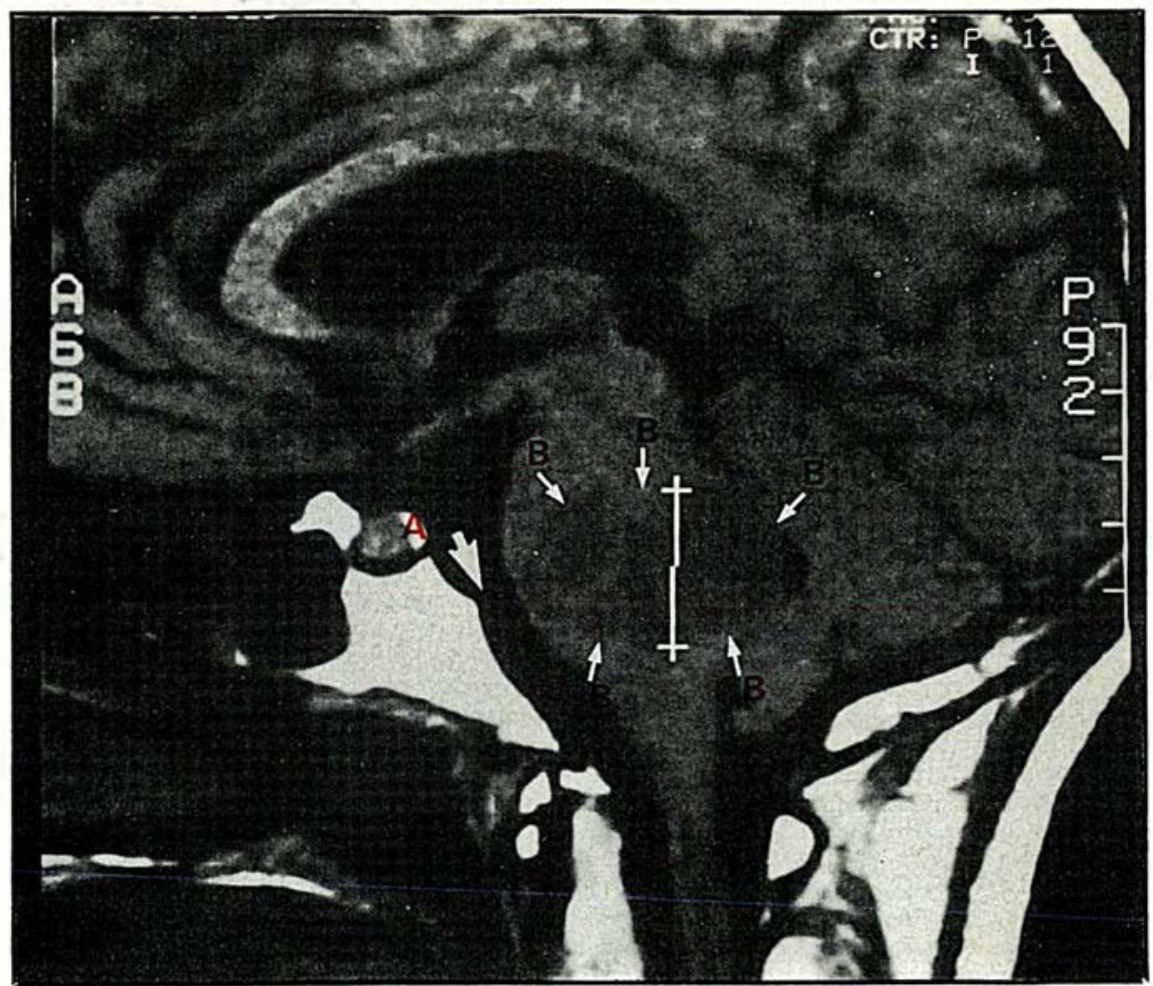

Figure I. Tumeur kystique du tronc cérébral: glioblastome probable. (Saturation partielle, PS 600/25 ms) : séquence pondérée en $T_{I}$. Coupe sagittale : absence de signal émis par la corticale osseuse (A). Volumineux processus expansif hypointense du tronc cérébral (13) refoulant le $4^{e}$ ventricule (cliché CIERM).

l'IRM précisera avec exactitude la présence et surtout l'extension. Dans la fosse postérieure (figure $I$ ) a u niveau du tronc cérébral, l'absence de signal émis par la corticale osseuse élimine les artéfacts fréquents en TDM. L'excellente visualisation tumorale ainsi obtenue, jointe à l'obtention de coupes coronales et sagittales des trois étages du tronc cérébral sur une seule coupe, permet de préciser avec exactitude l'extension des lésions : au niveau du cervelet où elle s'avère deux fois plus fréquente qu'au scanner, encore mieux au niveau de la moelle cervicale (où $83 \%$ sont sous-estimées en TDM) et de la médulla (64\% des cas sous-estimés) [4].

Par contre, l'augmentation globale des paramètres tumoraux rend difficile leur individualisation histologique. De petites variations du $T_{\text {I }}$ en fonction de la malignité des gliomes permettront probablement leur caractérisation ultérieure. D'autre part, l'œdème péritumoral, bien individualisé en TDM, présente en IRM un signal équivalent, peu discernable de la masse tumorale, et limite donc la précision du bilan d'extension; des produits de contraste tels que le Gadolinium-DTPA sont actuellement à l'étude [5] : agissant par diminution des temps de relaxation tumoraux, ils permettent une augmentation du contraste tumoral et son individualisation par rapport à l'œdème et à la nécrose. De demi-vie courte, on ne leur connait pas actuellement d'effets secondaires aux doses utilisées.

Les tumcurs extra-axiales sont de mise en évidence plus variable. Assez difficile pour les méningiomes : d'aspect caractéristique en TDM, leur mise en évidence en IRM requiert l'utilisation de 
séquences particulières. Les calcifications n'émettant aucun signal sont de mise en évidence aléatoire, sauf si elles sont volumineuses; par contre l'œdème, aux $T_{I}$ et $T_{2}$ plus longs que la tumeur, est bien discerné. Pour les méningiomes, la spécificité et la sensibilité de la TDM et de l'IRM apparaissent donc largement équivalentes.

A l'opposé, la sensibilité de l'IRM apparaît excellente dans l'exploration des neurinomes du nerf acoustique $[6,7,8]$. L'IRM permet d'une part d'analyser la composante anatomique de la tumeur par son signal intense, homogène à limites nettes en spin-écho et dans les trois dimensions, ainsi que son effet de masse sur le tronc cérébral en technique d'IRM. Elle permet d'autre part d'analyser une composante fonctionnelle sous forme d'un signal cérébelleux ou tronculaire témoignant d'une souffrance tissulaire. L'absence de signal vasculaire évite les "faux négatifs " en tomodensitométrie, liés à des boucles artérielles intra-canalaires.

L'utilisation des antennes de surface, le développement de champs magnétiques plus puissants améliorant le rapport signal bruit (RSB) et l'obtention d'une meilleure résolution spatiale, la réalisation de multi-coupes (permettant la visualisation constante des nerfs dans le conduit auditif interne) devraient permettre de détecter les petits neurinomes intra-canalaires et l'abandon de techniques invasives comme la cisterno-TDM.

La TDM à haute résolution avait révolutionné le diagnostic des lésions sellaires et supra-sellaires grâce à la réalisation des coupes coronales et sagittales par reconstruction. Si la TDM démontre avec constance la plupart des processus expansifs, l'IRM lui apparaît très supérieure $[9$, Io] dans la visualisation de ses rapports avec les éléments du tractus visuel éliminant le recours à la cisterno-TDM (voir figure 2). L'absence de signal vasculaire lié aux flux permet d'apprécier le refoulement de ces structures et surtout le siège de la carotide intra-caverneuse dispensant à l'avenir des artériographies carotidiennes pré-opératoires. Cependant, les microadénomes qui n'élargissent pas la glande pituitaire sont encore de détection difficile. La plupart des tumeurs présentent peu de modifications spécifiques de leurs signaux : le sang, la graisse, le cholestérol, le calcium qui modifient le signal IRM de façon caractéristique permettent cependant une caractérisation tissulaire dans certains cas. Comme au niveau canalaire, l'amélioration de la résolution spatiale, l'utilisation des antennes de surface et des produits de contraste modifieront probablement à l'avenir le pouvoir de détection et de caractérisation tumorale de l'IRM.

L'exploration des lésions démyélinisantes représente un des apports les plus spectaculaires de l'IRM [II, I2] puisque seulement I0\% des plaques détectées en IRM sont découvertes en TDM; les lésions sont en règle toujours plus étendues; les lésions de grade I et 2 ne sont pas vues en TDM. Sur le plan physiopathologique, le processus de démyélinisation libérant les protons liés aux lipides est responsable d'une augmentation des temps de relaxation par rapport au parenchyme cérébral. D'aspect le plus souvent arrondies, à bords nets, parfois linéaires dans la direction des fibres, les plaques de démyélinisation apparaissent ainsi caractéristiques par leur abondance, et leur distribution anatomique asymétrique dans la substance blanche périventriculaire, ou au niveau du corps calleux (figure 3). Aujourd'hui l'amélioration de la résolution spatiale et du RSB permet la détection des plaques au niveau médullaire en particulier cervical [ 13 ].

L'IRM semble aussi s'affirmer comme une excellente technique de visualisation des malformations artério-veineuses [14] identique, voire supérieure à la TDM : à condition toutefois d'atteindre un certain volume, la composante vasculaire apparaît sous forme serpigineuse avec absence de signal en cas de flux rapide habituel, tandis que l'utilisation de séquences adaptées peut faire apparaître un signal en $\mathrm{T}_{2}$ traduisant un flux plus lent artériel ou veineux.

Soulignons enfin le rôle potentiel de l'IRM puisque sa sensibilité s'avère très supérieure à la TDM dans les localisations cérébrales du lupus

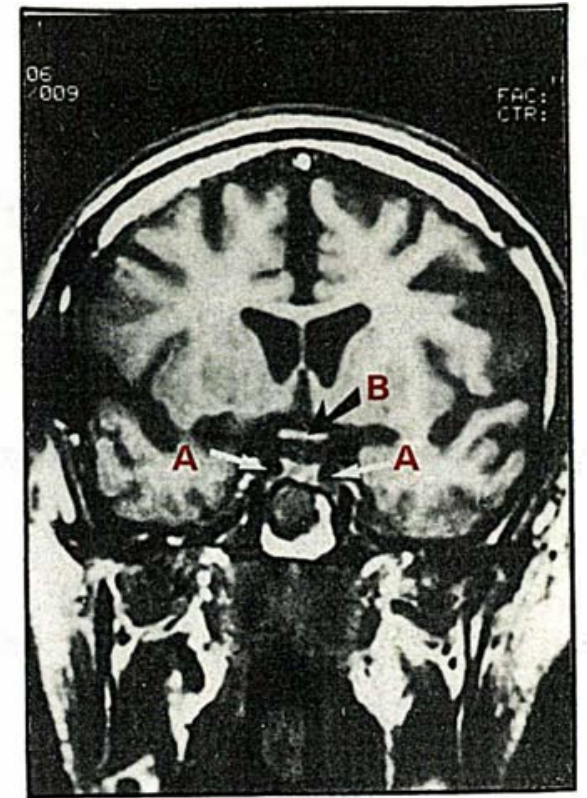

Figure 2. Région de la selle turcique en coupe coronale (PS600 ms). Noter l'absence de signal dans la carotide intracaverneuse (A) et l'exceptionnelle visibilité du chiasma optique (B) (cliché CIERM).

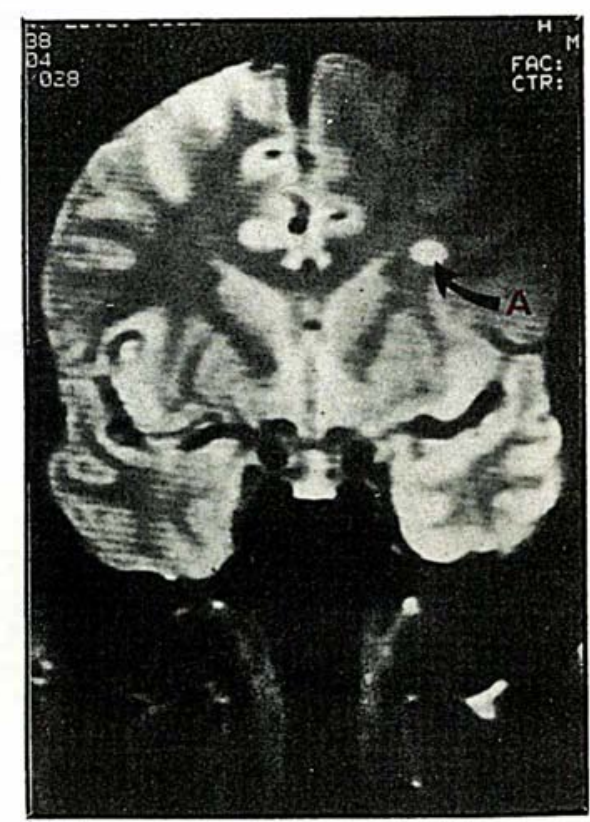

Figure 3. Sclérose en plaques. (SE 2500/40 ms) : aspect hyperintense d'une plaque (A) dans la substance blanche périventriculaire (cliché CIERM). 
REFERENCES

I1. Bories J, Carpena JP, Chiras J, Tamral T, Ibazizen MT. Nuclear magnetic resonance: first results in multiple sclerosis. 7 Neuroradiol 1984 ; I I : 307-14.

12. Runge YM, Price AC, Kirshner HS. Magnetic resonance imaging of multiple sclerosis: a study of pulse-technique efficacity. $A J R, 1984$; 143 : $1015-26$.

13. Maravilla KR, Weinreb JC, Suss R, Nunnally $\mathrm{RL}$. Magnetic resonance demonstration of multiple sclerosis plaques in the cervival cord. $A 7 R$, 1985; $144: 381-5$.

14. Kucharczyk W, Pleghos LL, Uske A, et al. Intracranial vascular malformations: $M R$ and $C T$ imaging. Neuroradiol $1985 ; 156: 383-9$.

15. Aisen AM, Gabrielsen TO, McCune WJ. MRI of systemic lupus erythematosus involving

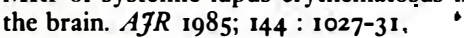

16. Aisen AM, Martel W, Gabriclsen TO, et al Wilson disease of the brain: MR imaging. Radiology $1985 ;$ 157: $137-41$.

17. Comori JM, Grossman RI, Goldberg HI, Zimmerman RA, Bilaniuk LT. Intracranial hematomas: imaging by high-field MR. Radiology $1985 ; 157: 87-93$.

18. Bradlev WG, Schmidt PG. Effect of methemoglobin formation on the MR appearance of subarachnoid hemorrhage. Radiology 1985 ; 156 : 99:103.

19. Pojunas $\mathrm{K}$, Williams $\mathrm{AL}$, Daniels $\mathrm{DL}$, Haughton VM. Syringomyelia and hydromyelia: Magnetic resonance evaluation. Radiology 1984; $153: 679-83$.

20. Lee BCP, Zimmerman RD, Manning JJ, Deck MDF. MR Imaging of syringomyelia and hydromyclia. $A 7 R, 1985 ; 144: 1149-56$.

21. Dichiro G, Doppman JL, Dwyer AJ, Patronas NJ, Knop RH, Bairamian D. Tumors and arteriovenous malf ormations of the spinal "cord: assessment using MR. Radiology 1985; 156 : 689-97.

22. Spinos E, Laster DW, Moody DM, Ball MR, Witcof ski RL, Kelly DL. MR evaluation of Chiari I malformations at 0,15 T. $A 7 R$ 1985; 144 : I 143-8.

23. Edelman RR, Shoukimas GM, Stark DD, et al. High resolution surface coil imaging of lumbar disk disease. $A 7 R$ 1985; 144: $1123-9$.

24. Aguila L, Piraino D, Modic MT. The intranuclear cleft of the intervertebral disk: magnetic resonance imaging. Radiology $1985 ; 155: 155-8$.

25. Modic MT, Pauligek W, Weinstein MA. Magnetic resonance imaging of intervertebral disk disease: clinical and pulse sequence considerations. Radiology 1984; 152 : 103-1 1 .

26. Modic MT, Feiglin DH, Piraino DW Boumphrey F; Weinstein MA, Duchesneau PM. Vertebral osteomyclitis: assessment using MR. Radiology 1985; 157 : 157-66. érythémateux [15], (mais sans pouvoir encore évaluer la possibilité de réversibilité lésionnelle), ainsi que dans la maladie de Wilson [I6] avec une meilleure corrélation entre la clinique et la découverte des lésions des noyaux gris.

\section{Pathologie traumatique}

En ce qui concerne la pathologie traumatique, $[17,18]$ on doit distinguer l'exploration de l'encéphale et celle du rachis. En outre les problèmes sont différents à la phase aiguë et à la phase tardive.

Pour l'encéphale, en phase aiguë, la TDM reste l'examen de choix par sa rapidité et sa spécificité de détection des hématomes intra ou extra-cérébraux. Par contre, au stade subaigu ou chronique, l'isodensité progressive du sang par rapport au parenchyme cérébral le rend de détection difficile. L'IRM trouve là une nouvelle indication importante, illustrant le rôle complémentaire de ces deux techniques : en effet, les modifications biochimiques de l'hématome liées à l'apparition progressive de la méthémoglobine sont responsables d'un intense signal, quelle que soit la séquence utilisée (car le TI est court et le T2 est long). Cet aspect caractéristique persistant plusieurs mois améliore donc très nettement la détection et la surveillance des lésions chroniques. D'autre part, du fait de la résolution de contraste de IRM et de l'absence d'artéfacts osseux, insistons sur la très nette amélioration diagnostique à ce stade des accidents hémorragiques au niveau du tronc cérébral et médullaire, tandis que la vue tridimensionnelle facilite la détection des hématomes péritentoriels et sous-temporaux sans perte d'information liée à la reconstruction (figure 4).

Pour le rachis, en phase aiguë, les données de l'IRM semblent de premier plan : visibilité dans les trois plans de la moelle et de ses enveloppes, sans nécessité d'opacification intrathécale supprimant le risque d'aggravation iatrogénique, possibilité de différencier une section médullaire complète d'une simple compression, voire d'une section incomplète : l'examen guidé par la cli- nique, éventuellement complété pour préciser une anatomie osseuse complexe, permettra de guider au mieux la thérapeutique interventionnelle agressive ou au contraire conservatrice.

\section{Pathologie}

médullorachidienne

La pathologie rachidienne comporte elle aussi une grande diversité étiologique : dégénérative, tumorale, infectieuse, congénitale. Le dénominateur commun de leur exploration radiologique restait jusqu'alors son caractère invasif, nécessitant le recours fréquent au scanner couplé à la myélographie (TDM-M).

Au niveau médullaire, l'IRM a ainsi apporté d'emblée de très grands espoirs et radicalement modifié l'approche diagnostique : la visibilité spontanée de la moelle (éliminant la nécessité d'opacification intrathécale), dans les trois plans et sur des grands segments permet un bilan diagnostique et d'extension précis, complet et non invasif, tandis que l'absence d'artéfact osseux transforme l'étude de la jonction

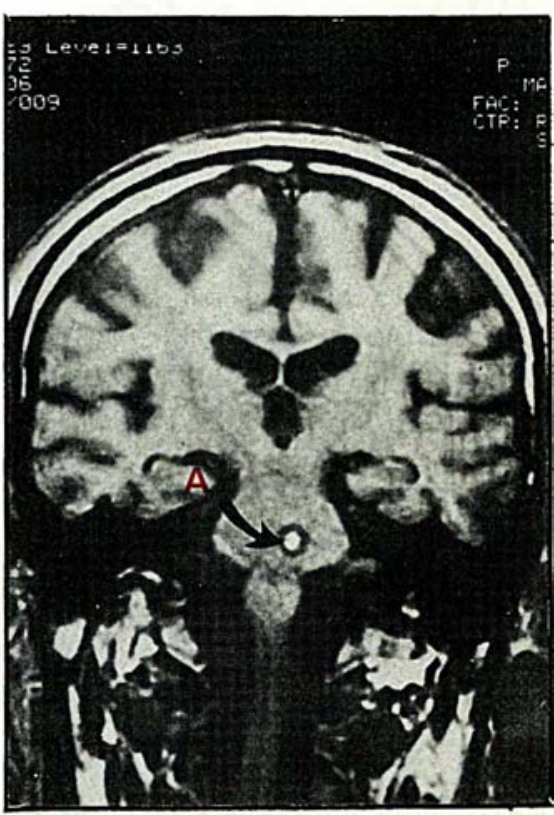

Figure 4. Hématome protubérantiel. PS $600 \mathrm{~ms}$. Petite zone hyperintense (A) protubérantielle, inchangée en séquence $S E 2000 / 40 \mathrm{~ms}$ : hématome protubérantiel (cliché CIERM). 


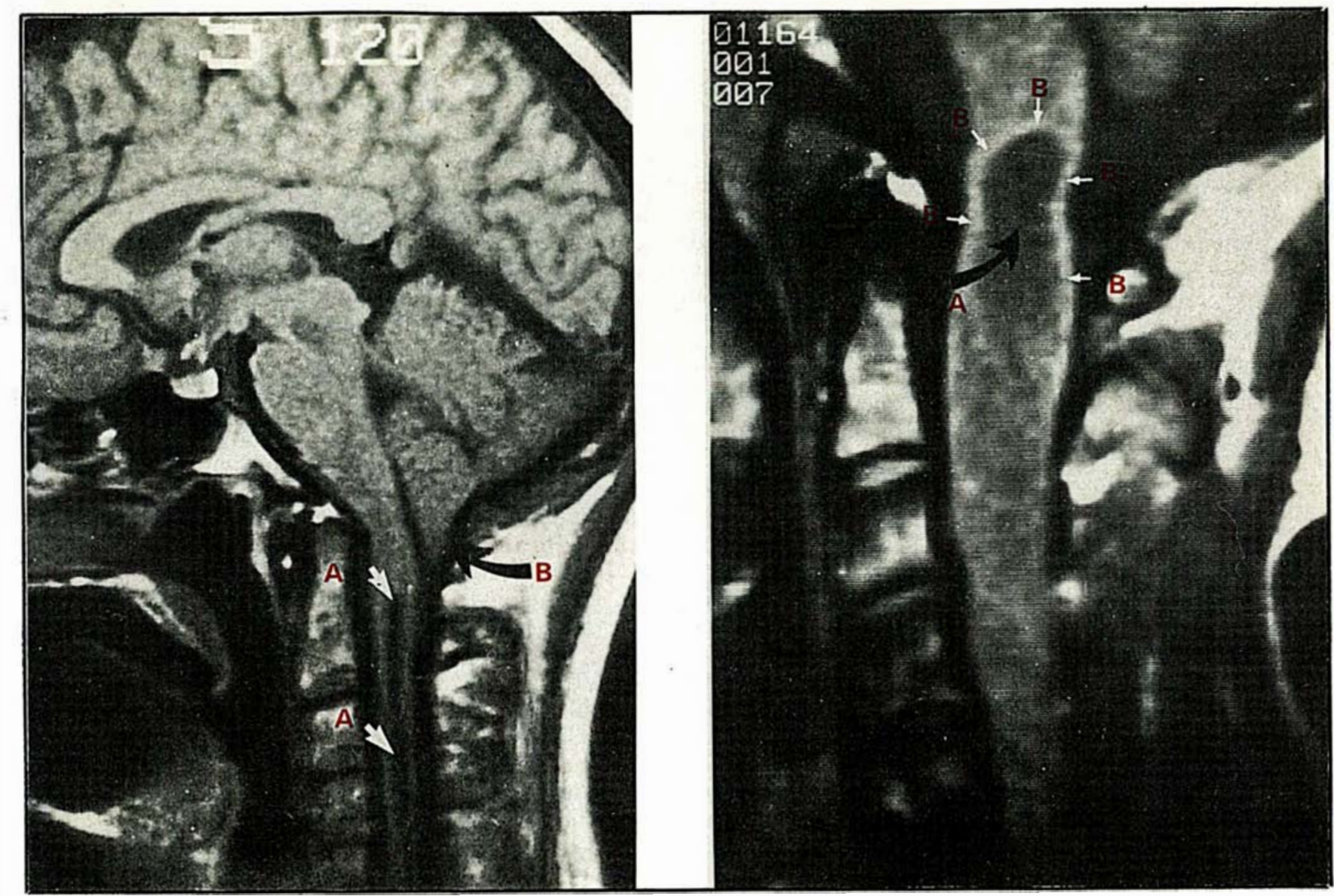

Figure 5. A gauche, Syringomyélie (PS $500 \mathrm{ms)}$ (coupe sagittale): fin kyste syringomyélique cervical (1 ); malformation de Chiari avec hernie amygdalienne (B); le $4^{e}$ ventricule est en place. A droite, tumeur kystique cervicale (coupe coronale): grosse moelle avec volumineux kyste (A) et hyperintensité tumorale périkystique (B) (cliché CIERM).

cranio-vertébrale. La longueur de l'examen, nécessitant la très bonne coopération du malade pose en règle peu de problèmes même en, pédiatrie, quoiqu'une légère sédation s'avère parf ois nécessaire.

Après le premier enthousiasme lié aux qualitís iconographiques de cette technique, quelle est la place actuelle de l'IRM?

Elle est fondamentale dans l'exploration des "grosses moelles ": l'augmentation de volume médullaire, d'appréciation aisée sur la réduction de volume des espaces sousarachnoïdiens, associée aux modifications des temps de relaxation tumoraux permettent un bilan diagnostique et d'extension, aisé et en un seul temps.

Son apport est en outre évident dans l'analyse des kystes médullaires et surtout dans la différenciation syringomyélie-tumeurs kystiques [19-22]. Cette différenciation nécessitait jusqu'à présent l'utilisation d'un protocole lourd associant la myélographie à des examens tomodensitométriques répétés sur 24 heures pour juger de la contamination de la cavité syringomyélique par le métrizamide, l'aspect du kyste tumoral ne se modifiant pas en règle. En IRM, l'aspect du kyste est souvent peu différenciable, voisin de celui du liquide céphalo-rachidien. Par contre, l'allongement des temps de relaxation de la portion périkystique tumorale par rapport à la moelle normale permet sa distinction aisée de la cavitation syringomyélique, kystique pure, et son bilan d'extension en un seul examen IRM ( figure 5).

Dans la syringomyélie, les coupes sagittales démontrent dans le même temps les malformations de Chiari [22], (hernies amygdaliennes et malpositions du $4^{\mathrm{e}}$ ventricule), fréquemment associées et de mise en évidence plus ou moins aisée en TDM-M (figure 5). Ce bilan particulièrement positif doit cependant être modulé : l'utilisation des antennes de surface, en particulier cervicales, améliorant le RSB et donc la qualité de l'imagerie, permet une 


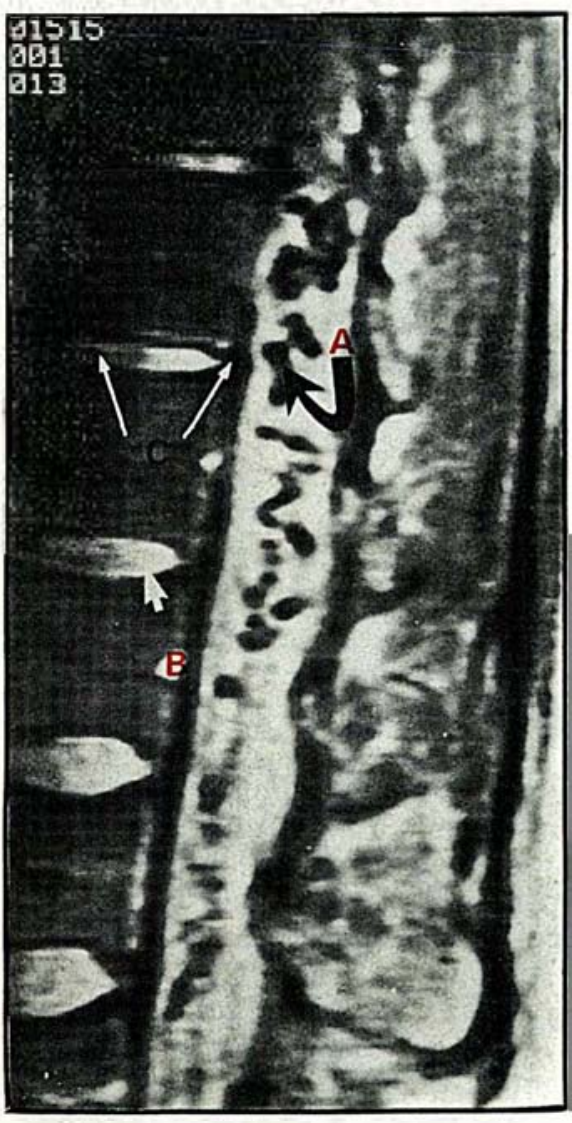

Figure 6. Malformation artérioveineuse médullaire. ( $S E 2500 / 40 \mathrm{~ms}$ ). Multiples images serpigineuses hypointenses (A). En avant, disques normaux: hyperintensité (B) du nucleus avec les hypointensités de sa division fibreuse antérieure et de l'annulus périphérique (C) (cliché CIERM). remarquable appréciation de l'extension céphalique y compris dans les moelles grêles. L'extension caudale reste cependant d'appréciation plus difficile, gênée en outre par les cyphoscolioses fréquentes et une moindre résolution axiale. D'autre part, un compromis doit être obtenu entre la finesse des coupes, la résolution spatiale et de contraste et la durée de l'examen.

L'IRM est donc l'examen de base et de première intention permettant souvent seule la découverte lésionnelle; une étude récente révèle cependant la possibilité de faux négatifs. Devant une clinique évocatrice, la TDM-M peut donc dans certains cas se discuter en seconde intention.

Les tumeurs intra-durales extra-médullaires (essentiellement les neurinomes et les méningiomes) bénéficient de même de l'IRM : visibilité tumorale spontanée, repérage aisé, même dans des localisations de diagnostic classiquement difficile comme la charnière cervico-occipitale. L'IRM pourrait également être importante dans les malformations artérioveineuses [2I] (figure 6) : le flux vasculaire rapide responsable d'une absence de signal permet la mise en évidence du nidus médullaire pouvant orienter l'artériographie médullaire; surtout, la thrombose modifie le signal qui devient hyperintense, permettant alors une surveillance simple et atraumatique de ces malades.

La supériorité de l'IRM sur le couple TDM-myélographie dans l'exploration de la ligne médiane trouve une indication de choix en pathologie congénitale : bien que gênée par les scolioses qui empêchent la visualisation monocoupe de la moelle, elle peut supprimer le TDM-M invasif en montrant de façon simple l'ectopie de la moelle, la densité hydrique et la localisation d'une méningocèle, les lipomes détectés facilement par leur hyperintensité constante ( $T_{1}$ court et $T_{2}$ long), et surtout les associations malformatives, y compris intra-crâniennes, par la vue d'ensemble qu'elle procure.

L'exploration de la pathologie ostéoarticulaire rachidienne paraît également prometteuse. L'étude de la morphologie discale permet en effet une nouvelle approche de son évolution dégénérative [23-25]: à l'état normal, le nucleus pulposus riche en eau apparaît sous forme d'un intense signal par rapport à l'annulus périphérique, plus fibreux (figure 6). Avec l'âge, la déshydratation et l'altération du collagène atténuent ce contraste signant la dégénérescence du disque. Celle-ci débute au niveau des éléments fibreux contenus dans le nucleus, puis s'étend à l'ensemble du disque.

La sensibilité de l'IRM apparaît ainsi supérieure à celle des autres techniques puisqu'un disque non dégénéré ne pouvant être hernié, la mise en évidence d'un disque de structure normale doit faire orienter le bilan vers une autre pathologie.

Par contre, en cas d'altération, l'utilisation de séquences spécifiques, en particulier à TE et TR longs, permettant un contraste spontané entre liquide céphalo-rachidien et disque appréciera une éventuelle hernie. Cependant, en cas de doute, en particulier dans les hernies latéralisées, des coupes axiales s'imposent, la valeur relative TDM-IRM restant encore alors discutée. En post-opératoire, la différenciation récidive discale-fibrose reste un problème délicat; l'IRM semble apporter ici des éléments positifs en visualisant un aspect variable : intensité basse avec effet de masse en cas de récidive discale, tandis que la cicatrice présente un signal de densité intermédiaire, hyperintense sur le premier écho, hypointense sur le second et sans effet de masse associé. Dans les spondylodiscites nécessitant un diagnostic et un traitement précoce, l'IRM semble s'imposer [26] par un aspect relativement caractéristique ne nécessitant que deux séquences : l'une pondérée en $T_{I}$, l'autre en $T_{2}$. La première permet de mettre en évidence l'hypointensité des disques et des corps vertébraux adjacents avec incapacité de discerner une limite entre le disque et le corps vertébral, tandis qu'une hyperintensité apparaît en T2. Les premières études réalisées montrent une sensibilité de $96 \%$, une exactitude diagnostique dans $94 \%$ des cas et une spécificité de $93 \%$. Plus précoce que la TDM et les clichés standard, elle apparaît aussi précoce que la scintigraphie, 
permettant une analyse topographique plus exacte, et un examen plus rapide. Surtout, elle ne semble pas précocement influencée par la mise en route du traitement antibiotique. Le retour à la normale est par contre plus lent. Le diagnostic différentiel pose en règle peu de problèmes : les processus dégénératifs ne modifient pas les signaux osseux adjacents, tandis que les métastases respectent en général les disques; la clinique guidant bien entendu toutes ces explorations.

De façon plus générale, pour l'exploration de l'os, seule la médullaire engendre un intense signal quelle que soit la séquence utilisée. Ce signal est en rapport avec la présence des cellules graisseuses et hématopoiétiques. Tout processus pathologique atteignant les cellules graisseuses se traduira donc par une diminution de leur signal; cet aspect est encore aspécifique. Les séries actuelles sont peu nombreuses. Cependant du fait de ses propriétés iconographiques, I'IRM semble déjà s'imposer en particulier dans les bilans d'extension tumorale cranio-caudal, au niveau intra-canalaire en cas de compression médullaire avec effacement des espaces sousarachnoïdiens, et surtout dans l'étude des envahissements musculaires périrachidiens.

\section{Conclusion}

Ainsi, la pathologie de la ligne médiane bénéficie au mieux de l'IRM. Celle-ci devrait s'imposer à l'avenir en première intention dans l'examen de la région sellaire, de la fosse postérieure et du tronc cérébral, ainsi que pour l'exploration de la moelle et du rachis. En pédiatrie en particulier, où cette localisation est fréquente, le caractère non invasif et non irradiant en fait une technique de choix. Le diagnostic des lésions démyélinisantes est radicalement transformé. L'exploration IRM en pathologie traumatique cérébrale est très utile à la phase tardive, tandis que celle des traumatismes rachidiens donne des informations importantes dès la phase aiguë. Il est prévisible que les plus grandes possibilités d'accès à l'IRM modifieront profondément à l'avenir les algorithmes neuroradiologiques

\section{Summary}

Magnetic resonnance imaging (MRI) is of considerable interest in neuroradiology. It is particularly useful in median line pathology, especially for the sella turcica, the posterior fossa and the brain stem as well as the spine and spinal canal, essentially in tumoral pathology but also in vascular, congenital or degenerative lesions. The diagnosis by MRI is particularly accurate in multiple sclcrosis. However, computerized tomography gives far more specific diagnostic information than MRP in acute trauma of the brain. MRI is useful in pediatry because it is non invasive and does not use X-rays or injections. MRI can be expected to greatly modify diagnostic strategy in neuroradiology.

\section{TIRÉS A PART}

N. Martin : Hôpital Beaujon, roo, boulevard du Général-Leclerc, 92 I 8 Clichy Cedex. 